Research Article

\title{
Joint Position and Velocity Estimation of a Moving Target in Multistatic Radar by Bistatic Range, TDOA, and Doppler Shifts
}

\author{
Lijuan Yang $(\mathbb{D}$, Huotao Gao $(\mathbb{D}$, Boya Li, Yunkun Yang, and Guobao Ru \\ Electronic Information School, Wuhan University, Wuhan 430072, China \\ Correspondence should be addressed to Huotao Gao; gaoght863@163.com
}

Received 5 June 2019; Revised 25 September 2019; Accepted 6 November 2019; Published 23 November 2019

Academic Editor: Felipe Cátedra

Copyright (c) 2019 Lijuan Yang et al. This is an open access article distributed under the Creative Commons Attribution License, which permits unrestricted use, distribution, and reproduction in any medium, provided the original work is properly cited.

\begin{abstract}
In this paper, an estimation algorithm for the position and velocity of a moving target in a multistatic radar system is investigated. Estimation accuracy is improved by using bistatic range (BR), time-difference-of-arrival (TDOA), and Doppler shifts. Multistatic radar system includes several independent receivers and transmitters of time synchronization. Different transmitters radiate signals of different frequencies, and receivers detect the Doppler shifts of the received signals. These estimation parameters, BR, TDOA, and Doppler shifts, are readily available. The proposed algorithm combines different estimated parameters and optimizes estimation accuracy by two-step weighted least squares minimisations (WLS). This estimation algorithm is analysed and verified by simulations, which can reach the Cramer-Rao lower bound (CRLB) performance under mild Gaussian noise when the measurement error is small. Numerical simulations also demonstrate the superior performance of this method.
\end{abstract}

\section{Introduction}

The classical problem of position and velocity estimation in the multistatic radar system receives great attentions in recent years due to its importance to detection, parameter estimation, tracking, surveillance, and navigation systems [1-3]. Generally, there are two modes for the multistatic radar system $[4,5]$, one is that a multistatic radar system consists of one transmitter and $\mathrm{N}$ receivers $\left(\mathrm{T}-\mathrm{R}^{\mathrm{N}}\right)[6]$ and the other is that the multistatic radar system which is composed of $\mathrm{M}$ identical transmitters and $\mathrm{N}$ identical receivers, radiating in a common coverage area $\left(\mathrm{T}^{\mathrm{M}}-\mathrm{R}^{\mathrm{N}}\right)$ [3] The multistatic radar system structure of $\mathrm{T}^{\mathrm{M}}-\mathrm{R}^{\mathrm{N}}$ has the advantages in antistealth and antidestruction. In this structure, transmitters radiate signals of different frequencies, and those signals are received by receivers to detect the Doppler shifts. More transmitters and receivers would obtain more estimation parameters. Therefore, the multistatic radar system offers more flexibility and better system robustness as well as improves estimation accuracy of position and velocity.
Several localization algorithms about the multistatic system, including radar and sonar, have been developed such as time-of-arrival (TOA) [7], time-difference-of-arrival (TDOA) [8, 9], Doppler shift [10, 11], bistatic range (BR) [2], angle-of-arrival (AOA) $[8,12]$, and received signal strength (RSS) [13]. In the multistatic system, AOA needs a complex estimation algorithm. BR is equivalent to ellipse localization, which is relied on the propagation time of the transmitted signals travelling from transmitters to the target and reflected back to receivers. The transmitters and receivers are located at different sites [14]. Every single BR can form an ellipse where the target lies on, and the corresponding transmitter and receiver serve as its foci. The intersection of multiple ellipses can determine the approximate position of the target. TDOA, which is the time difference of the signal reflected from the target to different receivers, induces a hyperbola locus for the target to be located, with the associated different receivers as its foci. The intersection of the hyperbola from multiple transmitter-receiver pairs gives the target position. Ellipse and hyperbola can be obtained based on time synchronization of transmitters and receivers. 
Doppler shifts can be detected through receivers. A moving target creates Doppler shifts which depend on the location and speed of a target. Thus, it can be exploited to improve the localization accuracy in the multistatic radar system [12], and then the accuracy of velocity estimation can be improved.

Single parameter can be used to estimate the position or velocity in some cases, but the accuracy needs to be improved. Several localization algorithms have been researched using two types of measurements, such as BR and Doppler shifts [15], BR and bearing measurements [16], and ellipse and hyperbola [17]. Also, there is an estimation algorithm using time delay, Doppler shifts, and AOA measurements [18]. Using two or three kinds of estimated parameters improves the position or velocity accuracy than only one estimated parameter.

In this paper, we present a joint position and velocity estimation algorithm, which uses Doppler shifts, BR, and TDOA, to estimate the position and velocity of a moving target. This algorithm is optimized based on two-step weighted least squares minimisations (WLS) [9, 18]. In the first step, a set of pseudolinear BR, TDOA, and Doppler shift equations are established by introducing nuisance parameters, rewriting pseudolinear equations to a linear equation that can utilize the calculation formula of WLS, and then the initial estimation of position and velocity is obtained. In the second step, the error term of the first-stage solution is estimated by using the relationship between initial estimated terms and nuisance variables. Similarly, error terms are calculated by WLS, and then the final estimation of position and velocity is obtained through subtracting the error terms from initial values. Simulation results show that it has a better localization and velocity accuracy not only than the method of ellipse and hyperbola but also than BR and Doppler shifts.

We shall use the common convention that bold upper and lower case letters denote matrix and column vector. $I_{m}$, $0_{m}$, and $0_{m, n}$ represent an $m \times m$ identity matrix, a zero vector with the length $m$, and $m \times n$ zero matrix, respectively. The notations $A^{T}$ and $A^{-1}$ denote transpose and inverse of matrix $A$. The $l_{2}$ norm is denoted by $\|*\|$. The symbol $\otimes$ stands for Kronecker products, and $a^{0}$ denotes the true value of the variable $\alpha$.

\section{Problem Formulation for the Multistatic Radar System}

We consider the localization of a moving target in a threedimensional (3D) scenario using the multistatic radar system network with $\mathrm{M}$ transmitters and $\mathrm{N}$ receivers, whose positions are denoted by $t_{i}=\left(x_{i}^{t}, y_{i}^{t}, z_{i}^{t}\right)^{T}, i=1,2, \ldots, M$ and $s_{j}=\left(x_{j}^{s}, y_{j}^{s}, z_{j}^{s}\right)^{T}, j=1,2, \ldots, N$. The target position $u^{0}=(x, y, z)^{T}$, and the target velocity $v^{0}=\left(v_{x}, v_{y}, v_{z}\right)^{T}$. The geometry structure of the multistatic radar system is shown in Figure 1.

Each transmitter radiates a signal of different frequency, and all receivers observe the signal through two paths, one is the direct propagation from the transmitter and the other is the indirect reflection of a moving target. Signals from the

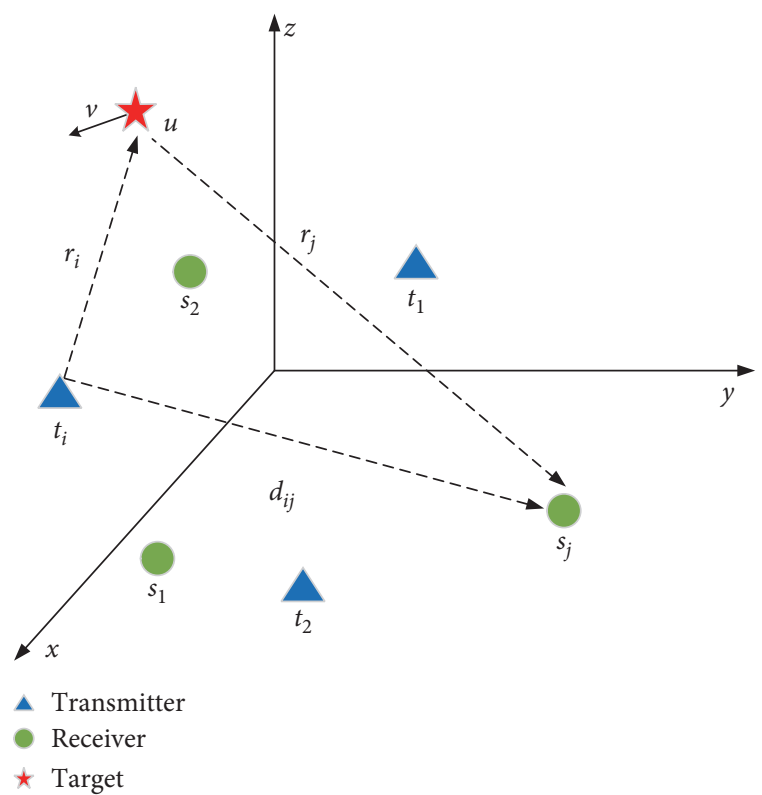

FiguRe 1: Geometry for multistatic radar.

two paths for each transmitter-receiver pair provide differential delay and Doppler shift measurements, where the latter comes from the target movement. The BRs $r_{i}+r_{j}$ are obtained based on the time synchronization of transmitters and receivers. TDOA is the time difference of signals reflected from the target to spatially separated receivers; it can be obtained due to the time synchronization of receivers. We will take advantage of these readily available parameters, BR, TDOA, and Doppler shift, to estimate the position and velocity of a moving target.

The range between the direct and the indirect signal from transmitter $i$ to receiver $j$ is given by

$$
r_{i j}^{0}=c \tau=r_{i}+r_{j}-d_{i j},
$$

where $c$ is the speed of signal transmission, $\tau$ is the differential delay between the direct and the indirect signal transmission, and $r_{j}=\left\|u^{0}-s_{j}\right\|, \quad r_{i}=\left\|u^{0}-t_{i}\right\|, \quad$ and $d_{i j}=\left\|t_{i}-s_{j}\right\| ;$ therefore, the BRs of the multistatic radar system are $r_{i j}^{0}+d_{i j}=c \tau+d_{i j}$.

Collecting the range measurements gives

$$
r=\left[r_{1}^{T}, r_{2}^{T}, \ldots, r_{M}^{T}\right]^{T}=r^{0}+\Delta r
$$

where $r_{i}=\left[r_{i 1}, r_{i 2}, \ldots, r_{i N}\right]^{T}=r_{i}^{0}+\Delta r_{i}, \Delta r_{i}=\left[\Delta r_{i 1}, \Delta r_{i 2}\right.$, $\left.\ldots, \Delta r_{i N}\right]^{T}$.

It is assumed that receiver $s_{1}$ is the reference receiver. After multiplying TDOA by signal propagation speed, the range difference between the receivers and $s_{1}$ can be expressed as $r_{j 1}^{0}=r_{j}-r_{1}, j=2,3, \ldots, N$ and the measured version of $r_{j 1}^{0}$ is $r_{j 1}=r_{j 1}^{0}+\Delta r_{j 1}$, where $\Delta r_{j 1}$ is the additive noise. We can get the $N-1$ hyperbolic measurements in vector form as

$$
\widetilde{r}=\left[r_{21}, r_{31}, \ldots, r_{N 1}\right]^{T}=\widetilde{r}^{0}+\Delta \widetilde{r} .
$$

Doppler shift is defined as the rate of the change of total path length of the signal to the time because transmitters and receivers are stationary, and the true Doppler shift is 


$$
f_{i j}^{0}=\frac{1}{\lambda}\left(\frac{\mathrm{d} r_{i}}{\mathrm{~d} t}+\frac{\mathrm{d} r_{j}}{\mathrm{~d} t}\right)=\frac{f_{c i}}{c}\left(\rho_{u^{0} t_{i}}^{T} \nu^{0}+\rho_{u^{0} s_{j}}^{T} \nu^{0}\right)
$$

where $\rho_{u^{0} t_{i}}=\left(u^{0}-t_{i}\right) /\left\|u^{0}-t_{i}\right\|$ denotes a unit vector from $t_{i}$ to $u^{0}$ and $f_{c i}$ is the carrier frequency of transmitter $t_{i}$; the measured $f_{i j}^{0}$ is $f_{i j}=f_{i j}^{0}+\Delta f_{i j}$, where $\Delta f_{i j}$ is the additive noise. Collecting the Doppler measurements gets

$$
f=\left[f_{1}^{T}, f_{2}^{T}, \ldots, f_{M}^{T}\right]^{T}=f^{0}+\Delta f,
$$

where $f_{i}=\left[f_{i 1}, f_{i 2}, \ldots, f_{i N}\right]^{T}=f_{i}^{0}+\Delta f_{i}, \quad \Delta f_{i}=\left[\Delta f_{i 1}\right.$, $\left.\Delta f_{i 2}, \ldots, \Delta f_{i N}\right]^{T}$.

Defining $m=\left[r^{T}, \widetilde{r}^{T}, f^{T}\right]^{T}, m$ is a $(2 M N+N-1) \times 1$ measurement vector, the error vector $\Delta m=\left[\Delta r^{T}\right.$, $\left.\Delta \widetilde{r}^{T}, \Delta f^{T}\right]^{T}$ is assumed to be zero-mean Gaussian with covariance matrix $Q_{m}=\operatorname{diag}\left(Q_{r}, Q_{\tilde{r}}, Q_{f}\right)$, where $Q_{r}, Q_{\tilde{r}}$, and $Q_{f}$ are covariance matrices of $r, \tilde{r}$, and $f$. We will use BR, TDOA, and Doppler shifts to estimate the position and velocity of a moving target.

The proposed algorithm is optimized by two steps. First, get the initial estimates through establishing a series of pseudolinear equations which introduce nuisance parameters. Second, get the final estimates by exploring the estimation error and estimating the amount of correction to refine the solution.

2.1. First Step. We calculate the initial position and velocity by introducing nuisance parameters and establishing a set of pseudolinear equations about BR, TDOA, and Doppler shift.

Rearranging $\quad r_{i j}^{0}=r_{i}+r_{j}-d_{i j}, \quad$ substituting $r_{i j}=r_{i j}^{0}+\Delta r_{i j}$, and squaring both sides yield the elliptic measurement equations. It can be obtained that

$$
\begin{aligned}
2 r_{j} \Delta r_{i j}= & r_{i j}^{2}+2 r_{i j} d_{i j}+2 t_{i}^{T}\left(t_{i}-s_{j}\right)-2\left(t_{i}-s_{j}\right)^{T} u^{0} \\
& -2\left(r_{i j}+d_{i j}\right) r_{i} .
\end{aligned}
$$

Note that BR and ellipse localization will be used interchangeably in this paper.

Similarly, rearranging $r_{j 1}^{0}=r_{j}-r_{1}$, substituting $r_{j 1}=r_{j 1}^{0}+\Delta r_{j 1}$, and squaring both sides yield the hyperbolic measurement equations. It can be expressed as

$$
2 r_{j 1} \Delta r_{j 1}=r_{j 1}^{2}-s_{j}^{T} s_{j}+s_{1}^{T} s_{1}+2\left(s_{j}-s_{1}\right)^{T} u^{0}+2 r_{j 1}\left\|u^{0}-s_{1}\right\| .
$$

Note that TDOA and hyperbola will be used interchangeably in this paper.

Then, rearranging (4) and $f_{i j}=f_{i j}^{0}+\Delta f_{i j}$, it can be expressed as

$$
\begin{aligned}
& 2 c \Delta f_{i j} r_{j}+2 f_{c i} \rho_{u^{0} s_{j}}^{T} v^{0} \Delta r_{i j}=2 c f_{i j} r_{i j}+2 c f_{i j} d_{i j} \\
& \quad-2 f_{c i}\left(t_{i}-s_{j}\right)^{T} v^{0}-2 c f_{i j} r_{i}-2 f_{i j}\left(r_{i j}+d_{i j}\right) \rho_{u^{0} t_{i}}^{T} v^{0} .
\end{aligned}
$$

In (6)-(8), the second-order noise terms have been ignored, and the unknown vector in the first stage can be $\varphi_{0}=\left[u^{0 T}, r_{i}^{0},\left\|u^{0}-s_{1}\right\|, v^{0 T}, \beta_{i}^{0}\right]$, where $\beta_{i}^{0}=\rho_{u^{0} t_{i}}^{T} v^{0}$ which contains $2 M+7$ nuisance variables. Equations (6)-(8) can be rewritten in the matrix form as follows:

$$
B_{1} \Delta m=h_{1}-G_{1} \varphi_{0} .
$$

This is a typical equation that can be solved using WLS, where $h_{1}=\left[h_{1 r}^{T}, h_{1 \widetilde{r}}^{T}, h_{1 f}^{T}\right]^{T}, h_{1 r}=r_{i j}^{2}+2 r_{i j} d_{i j}+2 t_{i}^{T}\left(t_{i}-s_{j}\right)$, $h_{1 \tilde{r}}=r_{j 1}^{2}-s_{j}^{T} s_{j}+s_{1}^{T} s_{1}$, and $h_{1 f}=2 c f_{i j} r_{i j}+2 c f_{i j} d_{i j}$;

$$
\begin{aligned}
G_{1} & =\left[G_{1 r}^{T}, G_{1 \widetilde{r}}^{T}, G_{1 f}^{T}\right]^{T}, \\
G_{1 r} & =2\left[\left(t_{i}-s_{j}\right)^{T}, 0_{i-1}^{T}, r_{i j}+d_{i j}, 0_{2 M-i+4}^{T}\right], \\
G_{1 \widetilde{r}} & =2\left[\left(s_{1}-s_{j}\right)^{T}, 0_{M}^{T},-\left\|s_{1}-s_{j}\right\|, 0_{M+3}^{T}\right], \\
G_{1 f} & =2\left[0_{i+2}^{T}, c f_{i j}, 0_{M-i+1}^{T}, f_{c i}\left(t_{i}-s_{j}\right)^{T}, 0_{i-1}^{T}, f_{c i}\left(r_{i j}+d_{i j}\right), 0_{M-i}^{T}\right], \\
B_{1} & =\left[\operatorname{blkdiag}\left(B_{1 r}, B_{1 \widetilde{r}}\right), B_{1 f}\right], \\
B_{1 r} & =2 I_{M} \otimes \operatorname{diag}\left(r_{j}\right), \\
B_{1 \widetilde{r}} & =2 \operatorname{diag}\left(r_{j 1}\right), \\
B_{1 f} & =\left[\operatorname{diag}\left(2 f_{c i} P_{u^{0} s_{j}}^{T} v^{0}\right), 0_{M N, N-1}, 2 c I_{M} \operatorname{diag}\left(r_{j}\right)\right] .
\end{aligned}
$$

The first-step WLS solution is $[19,20]$

$$
\varphi_{1}=\left(G_{1}^{T} W_{1} G_{1}\right)^{-1} G_{1}^{T} W_{1} h_{1},
$$

where $W_{1}=B_{1}^{-T} Q^{-1} B_{1}^{-1}$ is the weighting matrix. Since the variable $B_{1}$ depends on the estimated position and velocity, however, the initial estimations are unknown in the beginning; hence, we can first set $W_{1}=Q_{m}^{-1}$ to produce an initial solution, then use the initial solution to recalculate $B_{1}$, and finally expected $W_{1}$ can be obtained. When the noise is small, the covariance matrix of $\varphi_{1}$ is approximately equal to $\operatorname{cov}\left(\varphi_{1}\right) \approx\left(G_{1}^{T} W_{1} G_{1}\right)^{-1}$.

2.2. Second Step. The error terms are estimated by using the relationship between the solution of the first stage and nuisance variables.

We denote the first-stage estimation as $\varphi_{1}=\left[\tilde{u}^{T}, \widetilde{r}_{i},\left\|\tilde{u}-s_{1}\right\|, \widetilde{v}^{T}, \widetilde{\beta}_{i}\right]=\varphi_{1}^{0}+\Delta \varphi_{1}$, where $\Delta \varphi_{1}=\left[\Delta \widetilde{u}^{T}\right.$, $\left.\Delta \widetilde{r}_{i}, \Delta\left\|\widetilde{u}-s_{1}\right\|, \Delta \widetilde{v}^{T}, \Delta \widetilde{\beta}_{i}\right], r_{i}=\widetilde{r}_{i}-\Delta r_{i}$

Rearranging the parameters of $\varphi_{1}$ and $\Delta \varphi_{1}$, we get the equations about the nuisance variables and the estimation values from the first step:

$$
\begin{aligned}
2 \widetilde{r}_{i} \Delta \widetilde{r}_{i}=\widetilde{r}_{i}^{2}-\widetilde{u}^{T} \widetilde{u}-t_{i}^{T} t_{i}+2 t_{i}^{T} \widetilde{u}+2\left(\widetilde{u}-t_{i}\right)^{T} \Delta u & \\
2\left\|\widetilde{u}-s_{1}\right\| \Delta\left\|\widetilde{u}-s_{1}\right\|= & \left\|\widetilde{u}-s_{1}\right\|^{2}-s_{1}^{T} s_{1}-\widetilde{u}^{T} \widetilde{u}+2 s_{1}^{T} \widetilde{u} \\
& +2\left(\widetilde{u}-s_{1}\right)^{T} \Delta u
\end{aligned}
$$

Stacking (12)-(14) and putting them together yield the matrix form equation (15) which can be used to obtain estimation error terms by WLS: 


$$
B_{2} \Delta \varphi_{1}=h_{2}-G_{2} \Delta \vartheta
$$

where

$$
\begin{aligned}
\Delta \vartheta & =\left[\Delta u^{T}, \Delta v^{T}\right]^{T}, \\
B_{2} & =\left[B_{21}^{T}, B_{22}^{T}\right]^{T}, \\
B_{21} & =\left[\operatorname{blkdiag}\left[I_{3}, 2 r_{i}, 2\left\|\widetilde{u}-s_{1}\right\|\right], 0_{M+4, M+3}\right], \\
B_{22} & =\left[0_{M, 3}, \operatorname{diag}\left(\beta_{i}\right), 0_{M, 4}, \operatorname{diag}\left(r_{i}\right)\right], \\
h_{2} & =\left[0_{1,3}^{T}, h_{2 r}^{T}, h_{2 \widetilde{r}}^{T}, 0_{1,3}^{T}, h_{2 f}^{T}\right]^{T}, \\
h_{2 r} & =\widetilde{r}_{i}^{2}-\widetilde{u}^{T} \widetilde{u}-t_{i}^{T} t_{i}+2 t_{i}^{T} \widetilde{u}, \\
h_{2 \widetilde{r}} & =\left\|\widetilde{u}-s_{1}\right\|^{2}-s_{1}^{T} s_{1}-\widetilde{u}^{T} \widetilde{u}+2 s_{1}^{T} \widetilde{u}, \\
h_{2 f} & =\widetilde{r}_{i} \widetilde{\beta}_{i}-\widetilde{u}^{T} \widetilde{v}+t_{i}^{T} \widetilde{v}, \\
G_{2} & =\left[\left[-I_{3}, 0_{3,3}\right]^{T}, G_{2 r}^{T}, G_{2 \widetilde{r}}^{T},\left[0_{3,3},-I_{3}\right]^{T}, G_{2 f}^{T}\right], \\
G_{2 r} & =\left[-2\left(\widetilde{u}-t_{i}\right)^{T}, 0_{M, 3}\right], \\
G_{2 \widetilde{r}} & =\left[-2\left(\widetilde{u}-s_{1}\right)^{T}, 0_{1,3}\right], \\
G_{2 f} & =\left[-\widetilde{v}^{T},-\left(\widetilde{u}-t_{i}\right)^{T}\right] .
\end{aligned}
$$

The second-step WLS solution is

$$
\varphi_{2}=\Delta \vartheta=\left(G_{2}^{T} W_{2} G_{2}\right)^{-1} G_{2}^{T} W_{2} h_{2}
$$

where $\quad W_{2}=B_{2}^{-T} \operatorname{cov}\left(\varphi_{1}\right) B_{2}^{-1} . \quad \Delta u=\varphi_{2}(1: 3) \quad$ and $\Delta v=\varphi_{2}(4: 6)$. Under small noise conditions, we can get

$$
\operatorname{cov}\left(\varphi_{2}\right) \approx\left(G_{2}^{T} W_{2} G_{2}\right)^{-1}
$$

and $\widetilde{u}=\varphi_{1}(1: 3), \widetilde{v}=\varphi_{1}(4+M: 6+M)$ can be obtained from (11).

Finally, the estimations of position and velocity are

$$
\begin{aligned}
& u=\widetilde{u}-\Delta u, \\
& v=\widetilde{v}-\Delta v .
\end{aligned}
$$

\section{Performance Analysis}

The CRLB for estimating the unknown $\vartheta=\left[u^{T}, v^{T}\right]^{T}$ is equal to the inverse of the Fisher information matrix (FIM):

$$
\operatorname{CRLB}(\vartheta)=\left(\nabla^{T} Q_{m}^{-1} \nabla\right)^{-1}
$$

where $\nabla=[\partial \vartheta / \partial u, \partial \vartheta / \partial v]$.

The covariance matrix of $\vartheta$ is obtained from (21) as [1]:

$$
\operatorname{cov}(\vartheta)=\operatorname{cov}\left(\varphi_{2}\right)=\left(G_{3}^{T} Q_{m}^{-1} G_{3}\right)^{-1}
$$

where $G_{3}=B_{1}^{-1} G_{1} B_{2}^{-1} G_{2}$.

It can be obtained that $G_{3} \approx \nabla$ when $\Delta r_{i j} \ll r_{i j}$, $\Delta r_{j 1} \ll r_{j 1}, \Delta f_{i j} \ll f_{i j}, \Delta r_{i j} \ll\left\|u^{0}-s_{j}\right\|, \Delta r_{j 1} \ll\left\|u^{0}-s_{j}\right\|$, and $c \Delta f_{i j} \ll\left\|u^{0}-s_{j}\right\|$. The calculation of this follows similar procedures as in $[2,3,15,20-23]$.

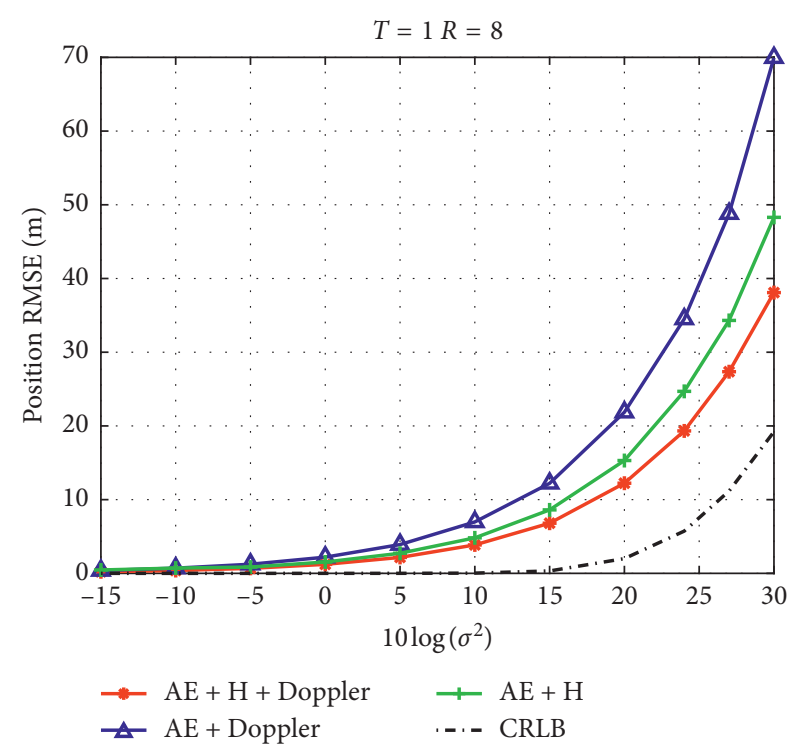

FIgURE 2: Comparison of the position RMSE of the proposed method with $\mathrm{AE}+$ Doppler, $\mathrm{AE}+\mathrm{H}$, and $\mathrm{CRLB}$ when $T=1, R=8$. The accuracy is lower as the noise power increases.

Consequently, we conclude from (20) and (21) that

$$
\operatorname{cov}(\vartheta) \approx \operatorname{CRLB}(\vartheta)
$$

when those small noise conditions are satisfied.

\section{Simulation Results}

The simulation scenario is as follows. A moving target is at $u^{0}=[1000,1500,3000]^{T} \mathrm{~m}$ and its velocity is $v^{0}=$ $[120,150,100]^{T} \mathrm{~m} / \mathrm{s}$. The multistatic radar system has $T=7$ transmitters and $R=8$ receivers. The transmitters are at $t_{1}=[3000,1000,0]^{T} \mathrm{~m}, t_{2}=[1000,1500,1000]^{T} \mathrm{~m}, t_{3}=$ $[3000,5000,2000]^{T} \mathrm{~m}, \quad t_{4}=[3500,2000,1000]^{T} \mathrm{~m}, t_{5}=$ $[3500,2000,0]^{T} \mathrm{~m}, \quad t_{6}=[0,0,0]^{T} \mathrm{~m}$, and $t_{7}=[-1000$, $3000,0]^{T} \mathrm{~m}$. The receivers' position are at $s_{1}=$ $[0,5000,0]^{T} \mathrm{~m}, \quad s_{2}=[5000,0,1000]^{T} \mathrm{~m}, \quad s_{3}=[-5000$, $0,1500]^{T} \mathrm{~m}, \quad s_{4}=[0,-5000,1000]^{T} \mathrm{~m}, \quad s_{5}=[0,0$, $1000]^{T} \mathrm{~m}, s_{6}=[0,1000,1000]^{T} \mathrm{~m}, s_{7}=[0,-2000,0]^{T} \mathrm{~m}$, and $s_{8}=[-5000,2000,0]^{T} \mathrm{~m}$. The signal propagation speed $c$ is $300000000 \mathrm{~m} / \mathrm{s}$. The carrier frequencies of the transmitted signals are $f_{c i}=[20,19,12,26,28,16,8] \mathrm{MHz}$. The covariance matrix $Q_{m}$ of BR, TDOA, and Doppler shift measurements is $Q_{m}=\sigma^{2} \operatorname{blkdiag}\left(I_{M}, I_{N-1}, 10^{5} I_{M}\right)$, $\sigma$ represents the level of noise. The localization and velocity accuracy are assessed via root-mean-square error (RMSE). The results in Figures $2-8$ are obtained from Monte Carlo simulations of 5,000 ensemble runs. We use CRLB as a benchmark for performance evaluation.

We compared performance of the proposed method with the CRLB and two kinds of algorithms, one is the hybrid of ellipse and hyperbola algorithm and the other is the algorithm of joint BR and Doppler shifts. The comparison algorithms were shown to outperform the same kind of algorithms. Simulation results are summarised in Figures 2-8, in which the curve marked with circle 


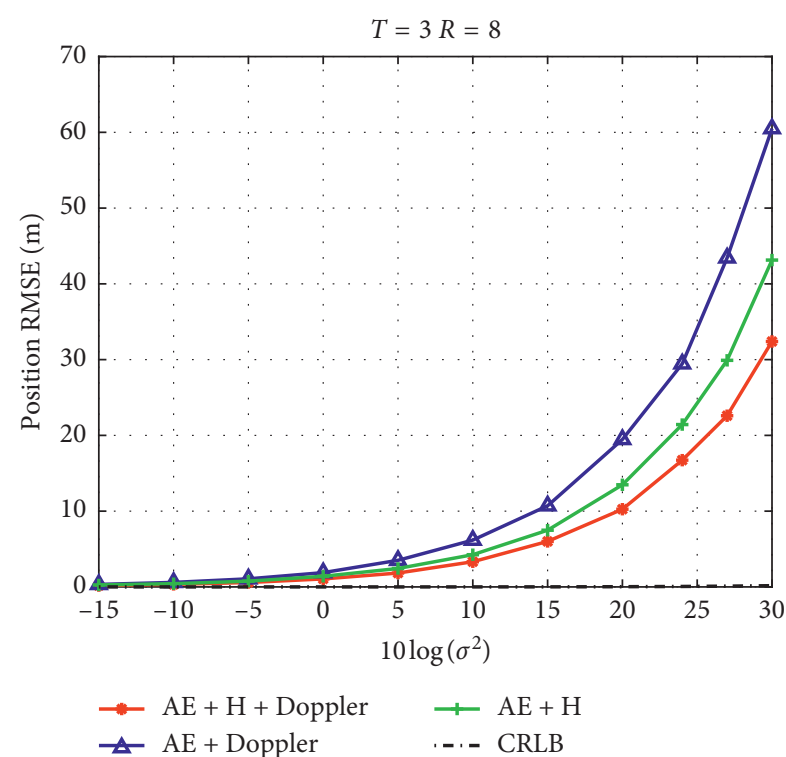

FIgURE 3: Comparison of the position RMSE of the proposed method with $\mathrm{AE}+$ Doppler, $\mathrm{AE}+\mathrm{H}$, and $\mathrm{CRLB}$ when $T=3, R=8$. The CRLB is close to zero, but it is going up as the noise power increases.

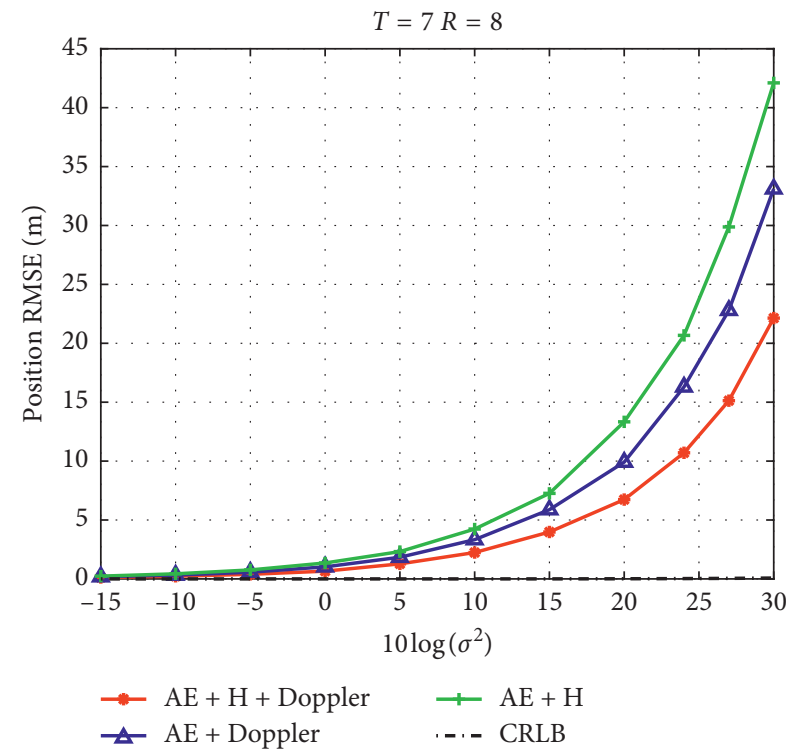

FIgURE 4: Comparison of the position RMSE of the proposed method with $\mathrm{AE}+$ Doppler, $\mathrm{AE}+\mathrm{H}$, and $\mathrm{CRLB}$ when $T=7, R=8$. The CRLB is close to zero, but it is going up as the noise power increases.

corresponds to the hybrid of ellipse, hyperbola, and Doppler shifts $(\mathrm{AE}+\mathrm{H}+$ Doppler), and curves marked with triangle and plus correspond to the joint ellipse and Doppler shifts $(\mathrm{AE}+$ Doppler $)$ and the joint ellipse and hyperbola $(\mathrm{AE}+\mathrm{H})$, respectively. The dotted line represents the CRLB under the corresponding setting.

In this paper, two kinds of modes are simulated, one is one transmitter and multiple receivers and the other is multiple transmitters and multiple receivers. The results

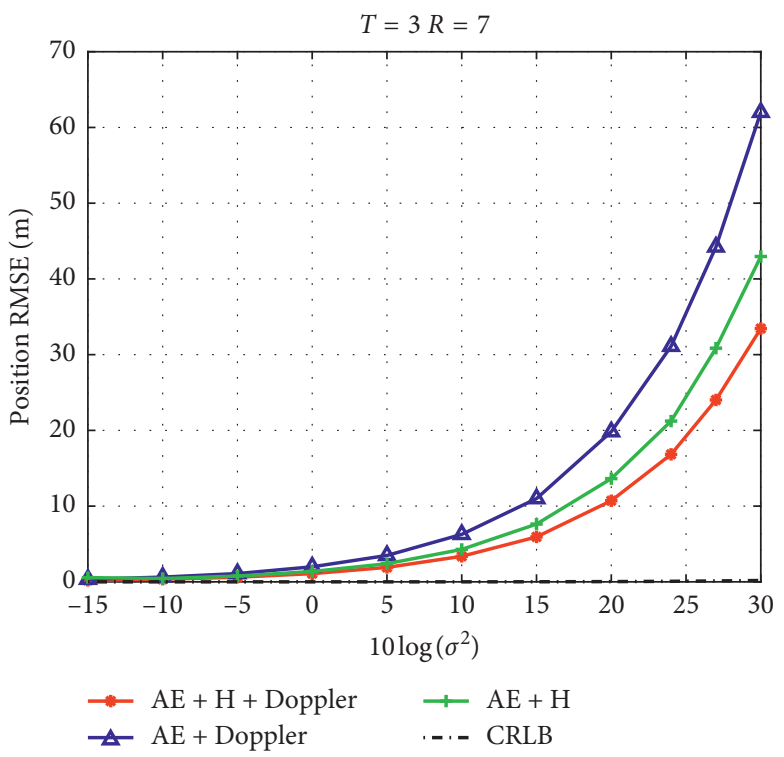

FIgURE 5: Comparison of the position RMSE of the proposed method with $\mathrm{AE}+$ Doppler, $\mathrm{AE}+\mathrm{H}$, and CRLB when $T=3, R=7$. The CRLB is close to zero, but it is going up as the noise power increases.

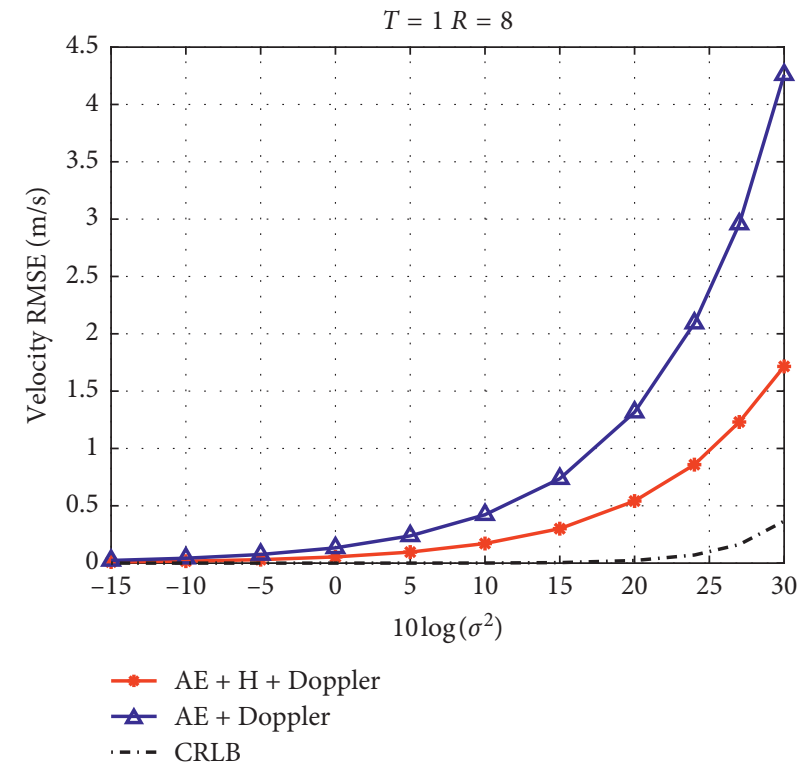

FIGURE 6: Comparison of the velocity RMSE of the proposed method with $\mathrm{AE}+$ Doppler and CRLB when $T=1, R=8$. The accuracy is lower as the noise power increases.

show that the proposed method is able to reach the CRLB when the measurement error is small. As is shown in Figures 2-5, under the condition of changing the number of transmitters or receivers, the proposed method has a better performance in the positioning compared to the hybrid of elliptic and hyperbolic method and the joint method of BR and Doppler shifts. Also, with the increase of $\sigma$, the position RMSE of the proposed method is obviously lower than that of the hybrid of elliptic and hyperbolic method and the joint method of BR and Doppler shifts. Then, in Figures 6-8, the 


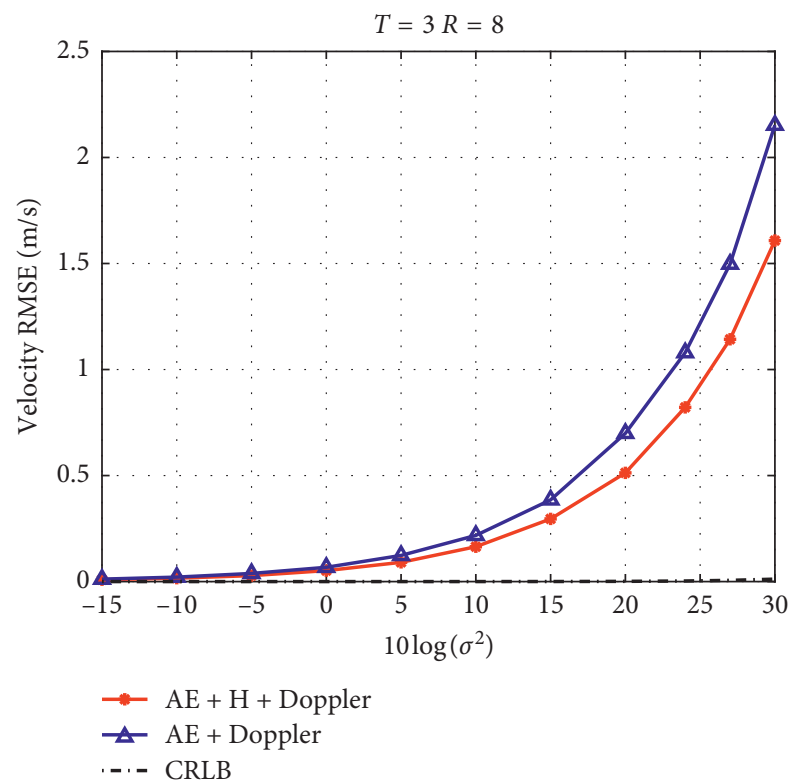

FIgURE 7: Comparison of the velocity RMSE of the proposed method with AE + Doppler and CRLB when $T=3, R=8$. The CRLB is close to zero, but it is going up as the noise power increases.

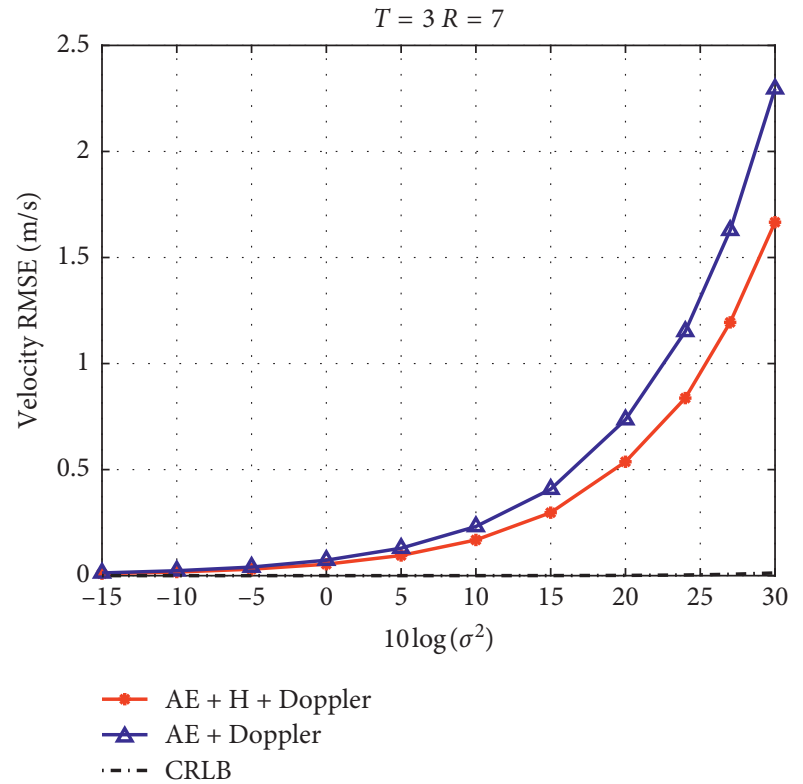

FIGURE 8: Comparison of the velocity RMSE of the proposed method with $\mathrm{AE}+$ Doppler and CRLB when $T=3, R=7$. The CRLB is close to zero, but it is going up as the noise power increases.

velocity RMSE is also analysed, and with the increase of $\sigma$, its gap between the proposed method and the hybrid of BR and Doppler shifts method becomes larger. The advantage of estimation performance is more obvious with the increase of measurement error. Moreover, when the number of receivers or transmitters is changed, the proposed algorithm has much better velocity accuracy than the joint method of BR and Doppler shifts. In Figures 3-5, 7, and 8, CRLB is close to zero when the number of transmitters and receivers is large. It is illustrated that the proposed algorithm has much better performance not only in the positioning but also in the velocity. Moreover, the estimation performance is affected by the number of transmitters and receivers, and estimation accuracy is improved when the number of transmitters and receivers is increased.

\section{Conclusion}

In this paper, we investigated the location and velocity of a moving target in the multistatic radar system by combining BR, TDOA, and Doppler shifts. This nonlinear estimation problem is solved by introducing nuisance variables and using two-step WLS to refine the estimate. It is found that the proposed method can obtain the CRLB accuracy over a small error region in the case of Gaussian noise. Simulation results have also shown its effectiveness. The proposed algorithm in this paper enriches the positioning methods of moving targets in the multistatic radar system.

\section{Data Availability}

MATLAB codes used in this study are available from the first author upon request (lcfylj@163.com).

\section{Conflicts of Interest}

The authors declare that there are no conflicts of interest regarding the publication of this paper.

\section{Acknowledgments}

This paper was supported by the National Natural Science Foundation of China (no.61671333), the Natural Science Foundation of Hubei Province (2014CFA093), the Fundamental Research Funds for the Central Universities (2042019K50264, 2042019gf0013), and the Fundamental Research Funds for the Wuhan Maritime Communication Research Institute (2017J-13).

\section{References}

[1] M. Malanowski and K. Kulpa, "Two methods for target localization in multistatic passive radar," IEEE Transactions on Aerospace and Electronic Systems, vol. 48, no. 1, pp. 572-580, 2012.

[2] R. Amiri, F. Behnia, and H. Zamani, "Asymptotically efficient target localization from bistatic range measurements in distributed MIMO radars," IEEE Signal Processing Letters, vol. 24, no. 3, pp. 299-303, 2017.

[3] P.-Z. Lei and X.-T. Huang, "Multistatic radar analysis based on ambiguity function and Cramér-Rao lower bounds," Journal of Central South University, vol. 21, no. 8, pp. 30923097, 2014.

[4] M. Abdul Hadi, R. Umar, M. Shoaib, M. Bilal, and K. Jamil, "Effectiveness of deghosting process for multi-target localization in multistatic passive radar," in Proceedings of the 2018 15th European Radar Conference (EuRAD), Madrid, Spain, September 2018.

[5] F. Sant, F. Pieralice, and D. Pastina, "Joint detection and localization of vessels at sea with a GNSS-based multistatic 
radar," IEEE Transactions on Geoscience and Remote Sensing, vol. 57, no. 8, pp. 5894-5913, 2019.

[6] J. Zhang, T. Jin, L. Qiu, W. Liu, and Z. Zhou, "Performance analysis for T- $\mathrm{R}^{\mathrm{N}}$ multistatic radar system," in Proceedings of the 2016 IEEE International Geoscience and Remote Sensing Symposium (IGARSS), Beijing, China, July 2016.

[7] N. H. Nguyen and K. Dogancay, "Optimal geometry analysis for multistatic TOA localization," IEEE Transactions on Signal Processing, vol. 64, no. 16, pp. 4180-4193, 2016.

[8] D. Torrieri, "Statistical theory of passive location systems," IEEE Transactions on Aerospace and Electronic Systems, vol. 20, no. 2, pp. 183-198, 1984.

[9] Y. T. Chan and K. C. Ho, "A simple and efficient estimator for hyperbolic location," IEEE Transactions on Signal Processing, vol. 42, no. 8, pp. 1905-1915, 1994.

[10] Y.-T. Chan and F. L. Jardine, "Target localization and tracking from Doppler-shift measurements," IEEE Journal of Oceanic Engineering, vol. 15, no. 3, pp. 251-257, 1990.

[11] I. Shames, A. N. Bishop, M. Smith, and B. D. O. Anderson, "Doppler shift target localization," IEEE Transactions on Aerospace and Electronic Systems, vol. 49, no. 1, pp. 266-276, 2013.

[12] K. Dogancay and H. Hmam, "Optimal angular sensor separation for AOA localization," Signal Processing, vol. 88, no. 5, pp. 1248-1260, 2008.

[13] A. N. Bishop and P. Jensfelt, "An optimality analysis of sensortarget geometries for signal strength based localization," in Proceedings of the 2009 International Conference on Intelligent Sensors, Sensor Networks and Information Processing ISSNIP, pp. 127-132, Melbourne, Australia, December 2009.

[14] L. Rui and K. C. Ho, "Elliptic localization: performance study and optimum receiver placement," IEEE Transactions on Signal Processing, vol. 62, no. 18, pp. 4673-4688, 2014.

[15] Y. Liu, L. Yang, and K. C. Ho, "Moving target localization in multistatic sonar by differential delays and Doppler shifts," IEEE Signal Processing Letters, vol. 23, no. 9, pp. 1160-1164, 2016.

[16] L. Rui and K. Ho, "Efficient closed-form estimators for multistatic sonar localization," IEEE Transactions on Aerospace and Electronic Systems, vol. 51, no. 1, pp. 600-614, 2015.

[17] Z. Qin, S. Wei, and J. Wang, "Efficient closed-form estimator for joint elliptic and hyperbolic localisation in multistatic system," Electronics Letters, vol. 54, no. 8, pp. 525-527, 2018.

[18] L. Yang, L. Yang, and K. C. Ho, "Moving target localization in multistatic sonar using time delays, Doppler shifts and arrival angles," in Proceedings of the 2017 IEEE International Conference on Acoustics, Speech and Signal Processing (ICASSP), pp. 3399-3103, New Orleans, LA, USA, June 2017.

[19] S. M. Kay, Fundamentals of Statistical Signal Processing: Estimation Theory, Prentice-Hall, Englewood Cliffs, NJ, USA, 1993.

[20] K. C. Ho and W. Xu, “An accurate algebraic solution for moving source location using TDOA and FDOA measurements," IEEE Transactions on Signal Processing, vol. 52, no. 9, pp. 2453-2463, 2004.

[21] S. Chen and K. C. Ho, "Achieving asymptotic efficient performance for squared range and squared range difference localizations," IEEE Transactions on Signal Processing, vol. 61, no. 11, pp. 2836-2849, 2013.

[22] X. Li, D. Wang, and X. Ma, "Three-dimensional target localization and Cramér-Rao bound for two-dimensional OFDM-MIMO radar," International Journal of Antennas and Propagation, vol. 2017, pp. 1-14, 2017.

[23] Y. Wang, W.-Q. Wang, and H. Shao, "Frequency diverse array radar Cramér-Rao lower bounds for estimating direction, range, and velocity," International Journal of Antennas and Propagation, vol. 2014, Article ID 830869, 15 pages, 2014. 


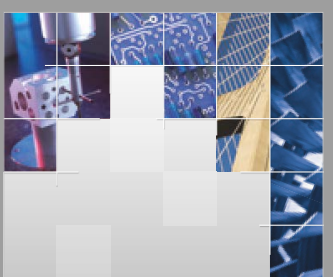

\section{Enfincering}
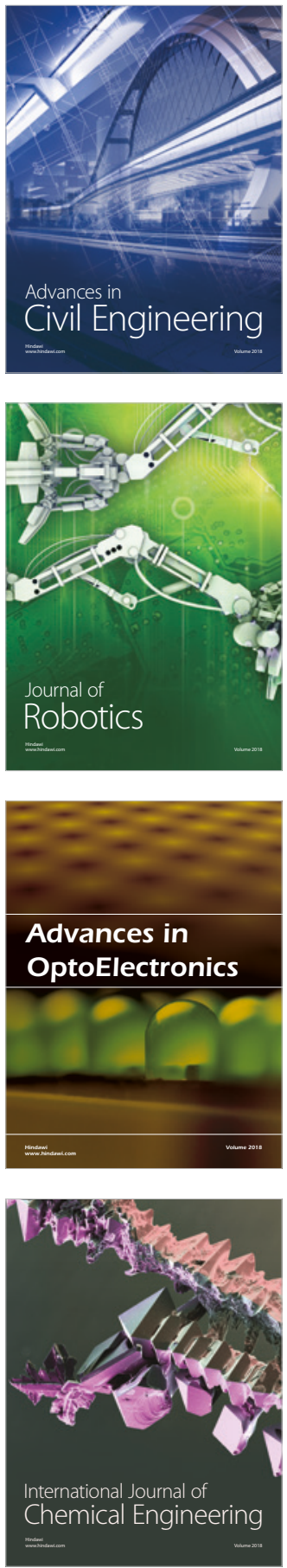

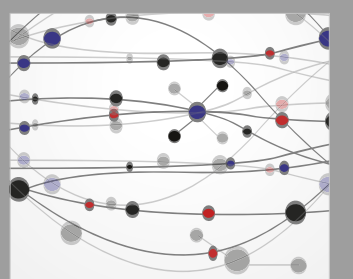

\section{Rotating \\ Machinery}

The Scientific World Journal

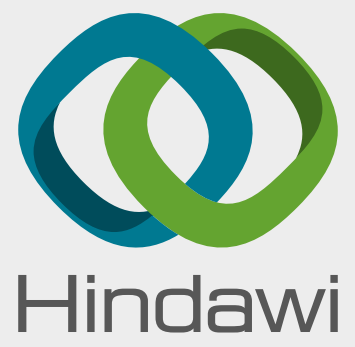

Submit your manuscripts at

www.hindawi.com
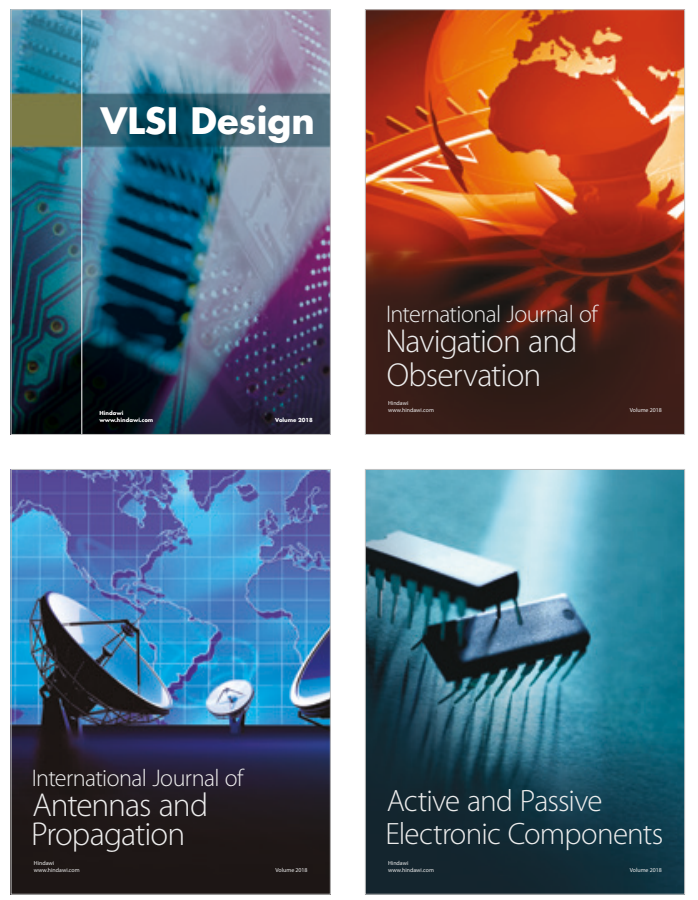
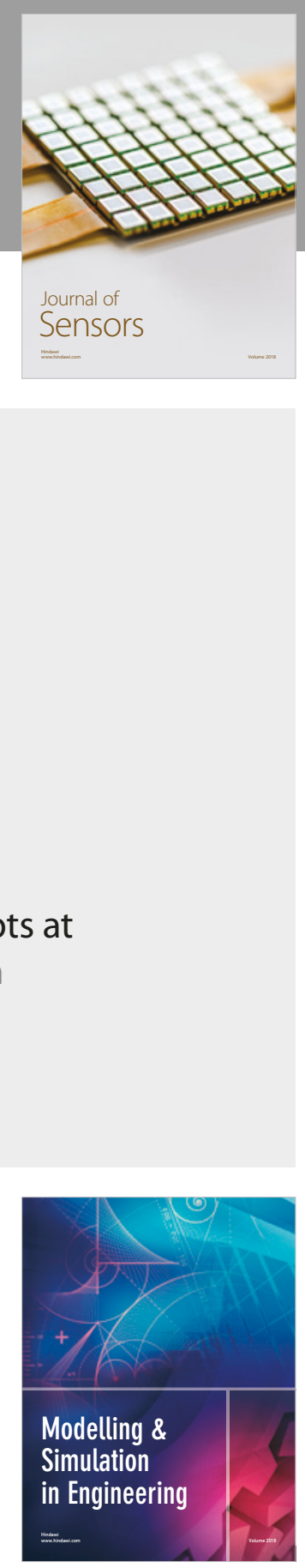

\section{Advances \\ Multimedia}
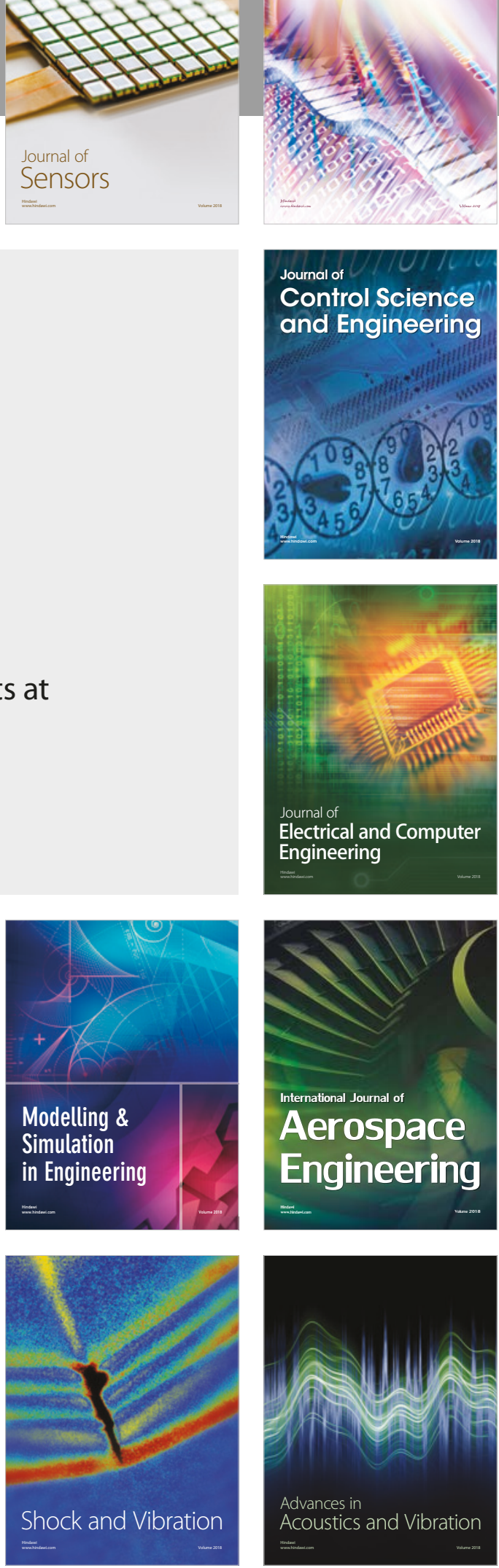